Article

\title{
Fumaric Acid Esters Do Not Reduce Inflammatory NF-кB/p65 Nuclear Translocation, ICAM-1 Expression and T-Cell Adhesiveness of Human Brain Microvascular Endothelial Cells
}

\author{
Axel Haarmann, Mathias Nehen, Annika Deiß and Mathias Buttmann* \\ Department of Neurology, University of Würzburg, Josef-Schneider-Str. 11, Würzburg 97080, \\ Germany; E-Mails: haarmann_a@ukw.de (A.H.); mathias.nehen@stud-mail.uni-wuerzburg.de (M.N.); \\ deiss_a@ukw.de (A.D.) \\ * Author to whom correspondence should be addressed; E-Mail: m.buttmann@ukw.de; \\ Tel.: +49-931-201-23777; Fax: +49-931-201-23488.
}

Academic Editor: Sven Meuth

Received: 1 June 2015 / Accepted: 10 August 2015 / Published: 13 August 2015

\begin{abstract}
Dimethyl fumarate (DMF) is approved for disease-modifying treatment of patients with relapsing-remitting multiple sclerosis. Animal experiments suggested that part of its therapeutic effect is due to a reduction of T-cell infiltration of the central nervous system (CNS) by uncertain mechanisms. Here we evaluated whether DMF and its primary metabolite monomethyl fumarate (MMF) modulate pro-inflammatory intracellular signaling and T-cell adhesiveness of nonimmortalized single donor human brain microvascular endothelial cells at low passages. Neither DMF nor MMF at concentrations of 10 or $50 \mu \mathrm{M}$ blocked the IL-1 $\beta$-induced nuclear translocation of NF- $\mathrm{kB} / \mathrm{p} 65$, whereas the higher concentration of DMF inhibited the nuclear entry of p65 in human umbilical vein endothelium cultured in parallel. DMF and MMF also did not alter the IL-1 $\beta$-stimulated activation of p38 MAPK in brain endothelium. Furthermore, neither DMF nor MMF reduced the basal or IL-1 $\beta$-inducible expression of ICAM-1. In accordance, both fumaric acid esters did not reduce the adhesion of activated Jurkat $\mathrm{T}$ cells to brain endothelium under basal or inflammatory conditions. Therefore, brain endothelial cells probably do not directly mediate a potential blocking effect of fumaric acid esters on the inflammatory infiltration of the CNS by T cells.
\end{abstract}

Keywords: blood-brain barrier; endothelial cells; multiple sclerosis; dimethyl fumarate; monomethyl fumarate; cell adhesion; NF-кB; p38 mitogen-activated protein kinase 


\section{Introduction}

Multiple sclerosis (MS) is a chronic degenerative autoimmune disease of the central nervous system (CNS). A disturbance of the blood-brain barrier (BBB) is central to its pathogenesis. The BBB is formed by highly specialized endothelial cells, controlling the exchange of solute, soluble factors and immune cells between blood and CNS tissue by complex molecular mechanisms [1]. A number of disease-modifying drugs approved for the treatment of patients with relapsing-remitting MS are supposed to reduce immune cell infiltration into the CNS. Natalizumab for example, a recombinant monoclonal IgG4 antibody against integrin $\alpha-4$, inhibits mononuclear leukocyte adhesion to activated CNS endothelium [2]. In addition, it was recently found to protect the paracellular endothelial barrier function by blocking integrin $\alpha-4$ expressed on CNS endothelial cells [3]. Another drug, the sphingosine-1-phosphate receptor (S1PR) modulator fingolimod, reduces T-cell infiltration into the CNS parenchyma by trapping $\mathrm{T}$ cells in peripheral lymphoid organs through functional antagonization of S1PR1 on lymphocytes [4]. In addition, it may decrease the extravasation of monocytes across CNS endothelial cells by activating endothelial S1PR5, thereby reducing endothelial NF- $\mathrm{BB}$ activation and inflammatory expression of adhesion molecules and chemokines [5].

Dimethyl fumarate (DMF), which is also approved for the disease-modifying treatment of patients with relapsing-remitting MS, is another drug that may reduce mononuclear immune cell infiltration of the CNS. Preventive treatment of C57BL/6 mice undergoing myelin oligodendrocyte glycoprotein (MOG) 35-55-induced experimental autoimmune encephalitis (EAE), an animal model of MS, with DMF or even more so with its primary metabolite monomethyl fumarate (MMF) was found to reduce infiltration of the spinal cord by CD3-positive T cells and Mac-3-positive mononuclear cells when analyzed 27 days after EAE induction [6]. In contrast, the same group did not observe a DMF-mediated reduction of mononuclear immune cell extravasation in the spinal cord 41 and 74 days after EAE induction [7]. The mechanisms by which fumaric acid esters (FAE) reduce mononuclear immune cell infiltration of the CNS in an earlier phase of EAE and possibly also in patients with MS are not entirely clear.

Modes of action may include FAE effects on immune cells, such as an induction of T-cell apoptosis, resulting in peripheral blood lymphopenia [8,9], or a reduced adhesive and migratory capacity of $\mathrm{T}$ lymphocytes at the BBB [10,11]. In addition, FAE may theoretically target endothelial cells at the inflamed BBB and inhibit immune cell extravasation by direct effects on endothelial cells. In human umbilical vein endothelial cells (HUVEC), DMF but not MMF blocked the adhesion of leukocytes in vitro by downregulating the inflammatory-inducible expression of the endothelial adhesion molecules ICAM-1, VCAM-1 and E-selectin [12,13], possibly by inhibiting the nuclear entry of activated $\mathrm{NF}-\kappa \mathrm{B} / \mathrm{p} 65$ resulting in reduced gene expression [14]. However, macrovascular HUVEC differ in many molecular and functional aspects from CNS microvascular endothelial cells [15]. Therefore, we set out to evaluate this potential mode of FAE action at the BBB in well characterized non-immortalized single donor human brain microvascular endothelial cells (HBMEC) at low passages. 


\section{Results}

\subsection{FAE Do Not Modulate NF-кB/p65 Nuclear Translocation or p38 MAPK Activation in HBMEC}

To comparatively evaluate effects of FAE on the inflammatory-stimulated nuclear translocation of NF-kB/p65 in HUVEC versus HBMEC, both cell types were cultured in parallel to subconfluency. Subsequently, cells were pretreated with DMF, MMF or DMSO solvent control for $24 \mathrm{~h}$ and then additionally stimulated with IL-1 $\beta$ for $1 \mathrm{~h}$ or left without further stimulation. In both HUVEC and HBMEC, IL-1 $\beta$ induced a comparable nuclear translocation of NF- $\mathrm{B} / \mathrm{p} 65$. However, while in HUVEC DMF at the higher tested concentration of $50 \mu \mathrm{M}$ clearly inhibited the nuclear entry of $\mathrm{NF}-\mathrm{kB} / \mathrm{p} 65$ (Figure 1a), which was in line with previously published results [14], neither DMF (Figure 1b) nor MMF (Figure 1c) blocked the nuclear entry of NF- $\mathrm{BB}$ in HBMEC compared to solvent control. Neither did DMF alter the basal or inflammatory activation of p38 MAPK in HBMEC (Figure 1d).
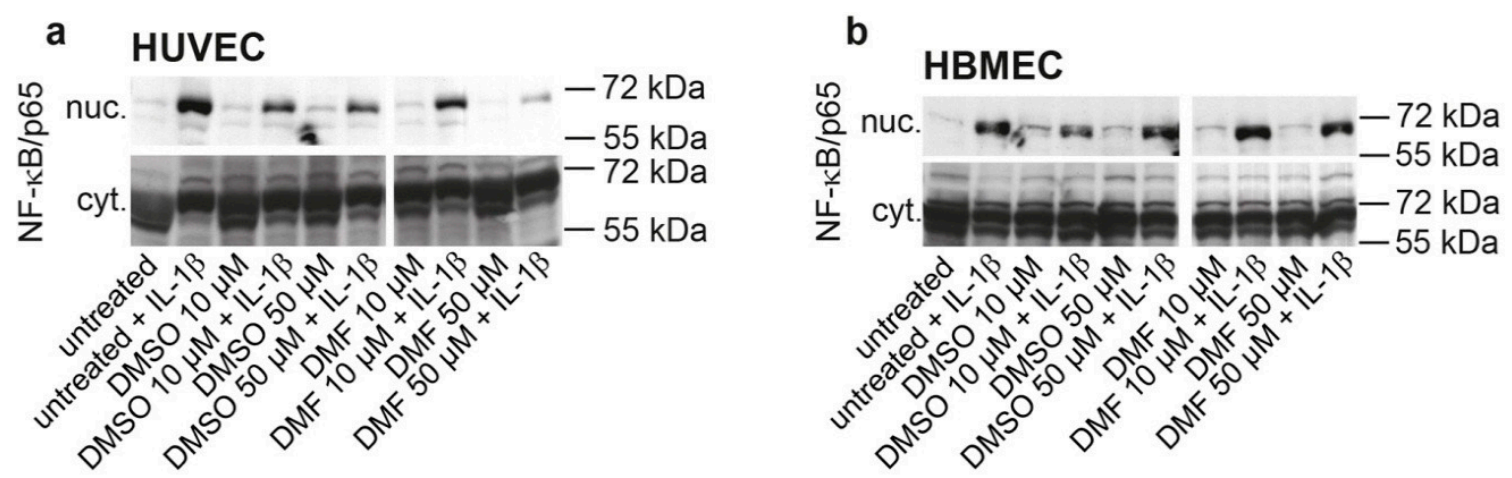

\section{c HBMEC}

\section{d HBMEC}
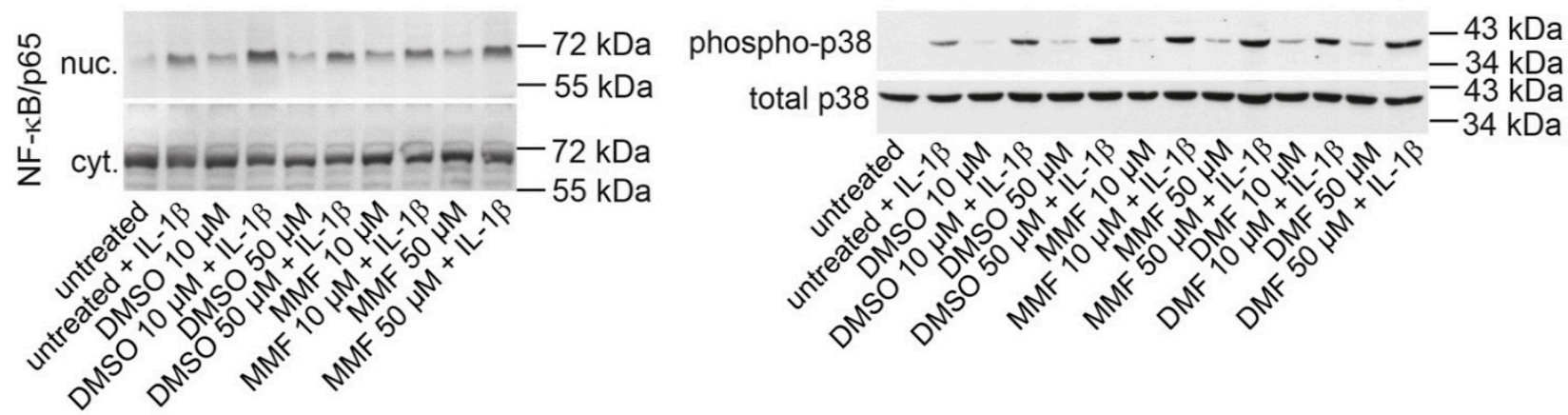

Figure 1. FAE do not modulate NF- $\mathrm{B}$ nuclear translocation or $\mathrm{p} 38$ MAPK activation in HBMEC. HUVEC (a) or HBMEC (b) were cultured to subconfluency in parallel and then left untreated or exposed to 10 or $50 \mu \mathrm{M}$ DMF, or to DMSO solvent control, corresponding to the DMSO concentrations present in the DMF samples, for $24 \mathrm{~h}$. Subsequently, cells were either not additionally stimulated or $10 \mathrm{ng} / \mathrm{mL}$ IL- $1 \beta$ were additionally added for one further hour. NF- $\mathrm{kB} / \mathrm{p} 65$ was then detected by Western blotting in nuclear (nuc.) and cytosolic (cyt.) cell protein extracts to investigate the nuclear translocation of NF- $\mathrm{kB} / \mathrm{p} 65$; (c) experiment in HBMEC as in (b), however using MMF instead of DMF; and (d) treatment of HBMEC in analogy to (b,c) as indicated, followed by Western blotting against phosphorylated and total p38 MAPK. Each panel representative of three independent experiments. 


\subsection{FAE Do Not Reduce Basal or Inflammatory-Inducible Expression of ICAM-1 in HBMEC}

Having observed that FAE did not reduce the IL-1 $\beta$-inducible nuclear translocation of NF- $\kappa \mathrm{B} / \mathrm{p} 65$ nor did they modulate the activation of p38 MAPK in HBMEC, we next evaluated whether DMF or MMF alter the basal or inflammatory-inducible expression of ICAM-1 as a prototypic NF- $\mathrm{BB}$-dependent adhesion molecule [16] with an important function for T-cell extravasation at the inflamed BBB [17]. Subconfluent HBMEC were pretreated with DMF, MMF or DMSO solvent control for $24 \mathrm{~h}$ and then additionally stimulated with IL-1 $\beta$ for another $24 \mathrm{~h}$ or did not receive additional stimulation. Neither DMF nor MMF changed the basal or inflammatory-inducible surface expression of ICAM-1 (Figure 2), which was in line with our previous result of a lack of NF- $\kappa$ B inhibition in HBMEC.

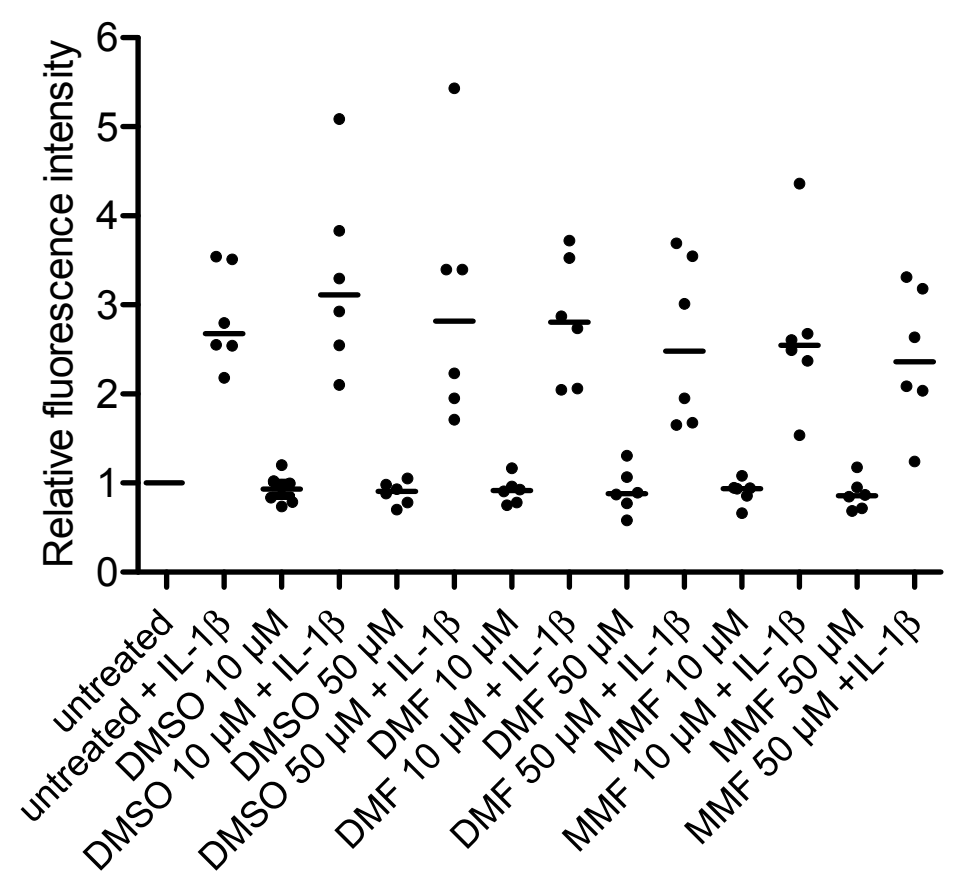

Figure 2. FAE do not reduce ICAM-1 expression on HBMEC under basal and inflammatory conditions. Subconfluent HBMEC were left untreated or exposed to DMF or MMF at concentrations of 10 or $50 \mu \mathrm{M}$, or to DMSO as a solvent control, at concentrations corresponding to the DMSO concentrations present in the FAE samples, for $24 \mathrm{~h}$. Subsequently, cells were not additionally stimulated or $10 \mathrm{ng} / \mathrm{mL}$ IL-1 $\beta$ were additionally added for another $24 \mathrm{~h}$. ICAM-1 surface expression after $48 \mathrm{~h}$ was assessed by flow cytometry. Geometric means of fluorescence intensities were compared to untreated cells and are reported as relative fluorescence intensities. Single values and medians of six independent experiments are shown.

\subsection{FAE Do Not Reduce T-Cell Adhesiveness of HBMEC}

In a last step, we assessed whether FAE modulate the T-cell adhesiveness of HBMEC under resting or inflammatory conditions. Confluent HBMEC were pretreated with DMF, MMF or DMSO solvent control for $24 \mathrm{~h}$ and then additionally stimulated with IL-1 $\beta$ or with TNF- $\alpha$ plus IFN- $\gamma$ for another $24 \mathrm{~h}$ or did not receive additional stimulation. Subsequently, adhesion of phorbol 12-myristate 13-acetate 
(PMA)-activated Jurkat T cells was assessed in a static adhesion assay. While inflammatory stimulation of HBMEC clearly increased their T-cell adhesiveness, neither DMF nor MMF reduced the adhesion of Jurkat $\mathrm{T}$ cells compared to solvent control under basal or inflammatory conditions (Figure 3).

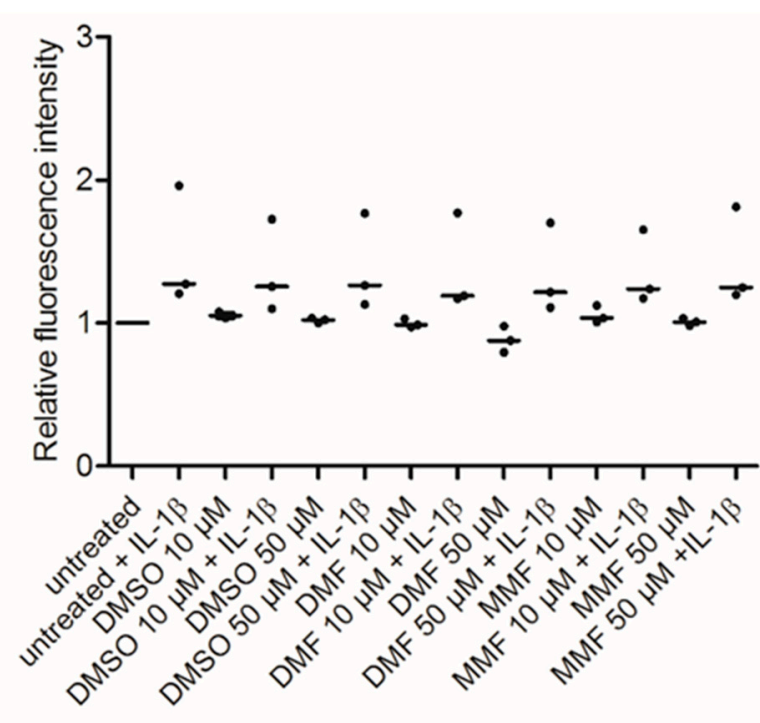

(a)

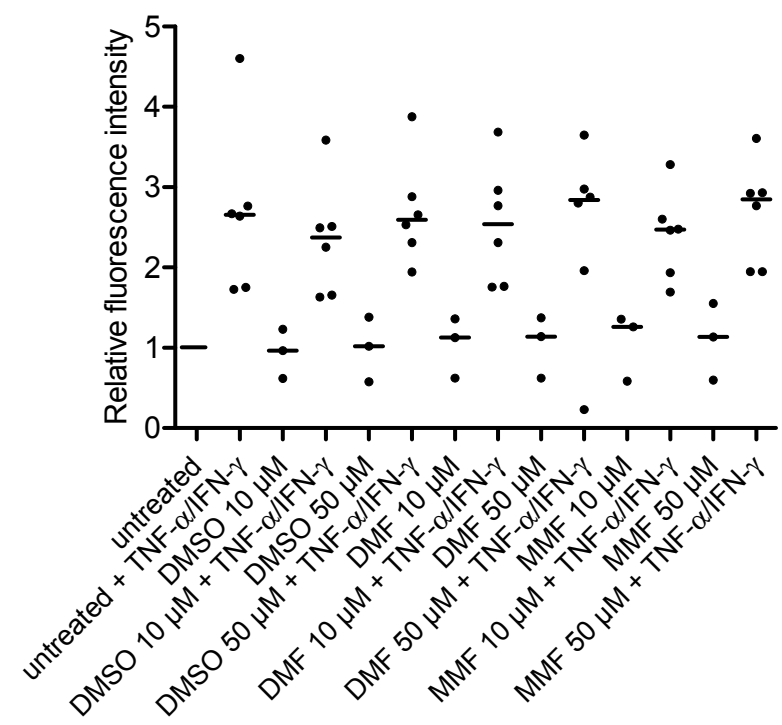

(b)

Figure 3. FAE do not reduce T-cell adhesiveness of HBMEC under basal and inflammatory conditions. Confluent HBMEC were stimulated in analogy to Figure 2, using IL-1 $\beta$ (a) or TNF- $\alpha$ plus IFN- $\gamma$ (b) as inflammatory stimuli. Subsequently, adhesion of calcein-labeled, PMA-activated Jurkat $\mathrm{T}$ cells was assessed in a static adhesion assay. Adhesion of Jurkat $\mathrm{T}$ cells, corresponding to the measured fluorescence intensities, is shown relative to untreated cells. Dots represent means of single independent experiments in octuplicates, bars represent medians.

\section{Discussion}

Here we report that FAE did neither reduce the inflammatory nuclear translocation of $\mathrm{NF}-\mathrm{kB} / \mathrm{p} 65$, nor the surface expression of ICAM-1, nor the T-cell adhesiveness of well characterized non-immortalized single donor HBMEC at early culture passages. This previously undescribed behavior of HBMEC is in contrast to findings in HUVEC, as independently reported by different groups and partially confirmed by ourselves in this study as a positive control [12-14]. Our in vitro results consistently argue that probably neither DMF, which after oral administration is not quantifiable in human plasma due to rapid hydrolysis [18] and therefore probably does not reach the human BBB at significant quantities, nor its immediate metabolite MMF directly act on endothelial cells at the inflamed BBB to reduce T-cell infiltration into the human CNS.

Our findings may be indirectly supported by an in vivo study that did not observe an antagonization of inflammatory claudin-5 downregulation at the BBB in DMF-treated C57BL/6 mice with $\mathrm{MOG}_{35-55}-\mathrm{EAE}$, arguing for a lack of stabilization of another aspect of BBB function by FAE under inflammatory conditions. Using immortalized hCMEC/D3 cells for in vitro experiments, however, both DMF and even more so MMF antagonized the TNF- $\alpha$-induced downregulation of claudin-5, occludin 
and zonula occludens-1 (ZO-1) mRNA and protein expression, which appeared to be in contrast to the in vivo results obtained by the same authors [19]. They concluded that their contradictory in vitro results may potentially be explained by the use of an immortalized cell line and that protection of the $\mathrm{BBB}$ is not a major mechanism in vivo. It should be noted, however, that in this study mice were analyzed for claudin-5 expression 72 days after EAE induction. The same authors had previously not observed an effect of DMF on CNS immune cell infiltration 41 and 74 days after MOG35-55-EAE induction [7], while reduced CNS immune cell infiltration was found 27 days after injection of $\mathrm{MOG}_{35-55}$ peptide [6]. It therefore seems conceivable that the chosen time point for analysis of claudin-5 expression was too late in the course of EAE to detect a protective effect of DMF treatment on BBB function. Such an effect still appears possible during earlier phases of the disease, which calls for additional investigations of the BBB during earlier phases of EAE.

A recent study exploring C57BL/6 mice undergoing experimental ischemia reperfusion injury, found a preservation of claudin-5 expression at the BBB along with reduced brain edema formation after oral pre-treatment of the mice with DMF [20]. Using the immortalized murine brain endothelial cell line bEND.3 for in vitro experiments, the same authors found a preservation of zonula occludens-1 (ZO-1) and VE-cadherin localization in oxygen-glucose deprived cells in the presence of DMF. Also employing bEND.3 cells, they observed reduced transendothelial migration of the human monocyte cell line THP-1 against CCL2 in the lower chamber of a transwell system after pre-treatment of the bEND.3 cells with DMF. They furthermore observed decreased ICAM-1, VCAM-1 and E-selectin mRNA expression in bEND. 3 cells after treatment with $50 \mu \mathrm{M}$ DMF for $6 \mathrm{~h}$. In their transmigration assays and mRNA expression studies, bEND.3 cells were unstimulated (apart from the presence of CCL2), leaving open the effect of DMF under oxygen-glucose deprived conditions and mechanistically posing the question whether $\mathrm{NF}-\mathrm{\kappa B}$ was activated in the unstimulated cells. In vitro results for MMF, the presumed pharmacologically active FAE, were not reported. Despite the congruency of the reported in vivo results, the bEND.3 in vitro findings should be interpreted with caution in our view, as this cell line was criticized by a number of authors for a lack of typical BBB properties [21-23], which corresponds to our own experience with bEND. 3 cells from various sources. In line with a limited barrier function of bEND. 3 cells, the authors of the discussed study did not observe a protection of the paracellular barrier function by DMF [20].

To study the organ- and potentially also species-specific behavior of highly specialized endothelial cells at the human BBB, the preserved differentiation and human source of the used cells is of crucial importance in our view. The non-immortalized human cells at early passages employed in this study are well characterized and were successfully used by us for a number of studies in the past. These cells respond to inflammatory stimulation, as demonstrated here by NF- $\kappa \mathrm{B} / \mathrm{p} 65$ nuclear translocation, p38 MAPK activation as well as increased ICAM-1 expression and increased T-cell adhesiveness. Furthermore, we additionally demonstrated a successful modulation of functional BBB properties by different pharmacological agents in these cells in the past $[3,24,25]$. We, therefore, believe that the reported non-responsivity of our HBMEC to FAE probably reflects the true behavior of HBMEC at the human BBB in vivo. The use of immortalized, instead of primary, $T$ cells for the adhesion assays represents a potential limitation of our study, although we successfully used them in a similar context in the past [25]. 


\section{Materials and Methods}

\subsection{Cell Culture}

Cryopreserved single donor primary HBMEC at passage 2 were purchased from Cell Systems Corp. (Kirkland, WA, USA) and cryopreserved single donor HUVEC at passage 1 from Promocell (Heidelberg, Germany). To exclude contamination by other cell types and to demonstrate the expression of tight junction-associated molecules, each HBMEC preparation was extensively characterized as previously described $[3,24,25]$. HBMEC purity was $>98 \%$ in all preparations. Mycoplasma contamination was excluded using a commercial PCR-based mycoplasma detection kit (PK-CA91; PromoKine, Heidelberg, Germany). Cells were grown on $2 \%$ gelatin-coated plates or flasks (all from Nunc, Roskilde, Denmark) at $37{ }^{\circ} \mathrm{C} / 5 \% \mathrm{CO}_{2}$, using M199 basal medium (Lonza, Cologne, Germany) supplemented with $10 \%$ fetal calf serum (FCS; Biochrom, Berlin, Germany), endothelial cell growth supplement (20 $\mu \mathrm{g} / \mathrm{mL}$; Sigma-Aldrich, Schnelldorf, Germany), heparin (100 $\mu \mathrm{g} / \mathrm{mL}$; Sigma-Aldrich), amphotericin B (250 $\mu \mathrm{g} / \mathrm{mL})$, gentamycin $(50 \mu \mathrm{g} / \mathrm{mL})$, penicillin $(50 \mathrm{U} / \mathrm{mL})$ and streptomycin $(50 \mu \mathrm{g} / \mathrm{mL}$; all from Invitrogen, Karlsruhe, Germany). Endothelial cells were used between passage 3 and 6 for experiments. Jurkat $\mathrm{T}$ cells (clone E6-1) were purchased from ATCC (Manassas, VA, USA) and cultured in RPMI1640 medium (Gibco, Life Technologies GmbH, Darmstadt, Germany) supplemented by 10\% FCS at $37{ }^{\circ} \mathrm{C} / 5 \% \mathrm{CO}_{2}$.

\subsection{Stimulation}

DMF and MMF were purchased from Sigma-Aldrich and dissolved in dimethyl sulfoxide (DMSO) to prepare $50 \mathrm{mM}$ stock solutions which were stored in aliquots at $-80^{\circ} \mathrm{C}$. For treatment of endothelial cells, FAE stock aliquots or DMSO as a solvent control were freshly diluted using M199 medium. Endothelial cells were treated with FAE at the indicated concentrations for the indicated durations. For inflammatory

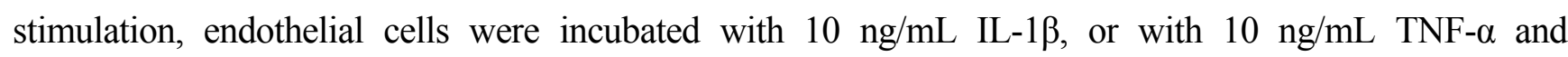
$100 \mathrm{IU} / \mathrm{mL}$ IFN- $\gamma$ (all from R\&D Systems, Karlsruhe, Germany) for the indicated durations. Jurkat T cells were activated by stimulation with $10 \mathrm{ng} / \mathrm{mL}$ PMA (Sigma-Aldrich) for $24 \mathrm{~h}$.

\subsection{Cell Protein Extracts and Western Blotting}

For Western blot experiments, endothelial cells were grown to subconfluency and stimulated as indicated. Nuclear and cytosolic protein extracts were prepared exactly as previously described [26]. Cell protein extracts were fractionated by 10\% SDS-PAGE and electroblotted onto nitrocellulose membrane. Ponceau staining served as a control for equal loading and complete protein transfer. Rabbit

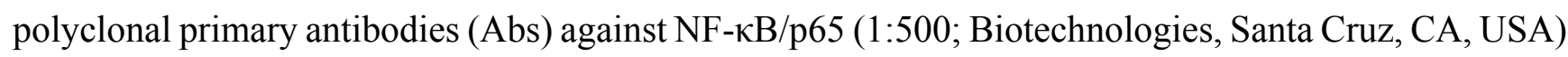
or phospho-p38 (1:1000; Cell Signaling, Cambridge, UK) were incubated over night at $4{ }^{\circ} \mathrm{C}$ and detected with an anti-rabbit-IgG peroxidase-coupled secondary $\mathrm{Ab}$ along with a standard enhanced chemoluminescence system. After peroxidase inactivation, membranes for $\mathrm{p} 38$ detection were reprobed with mouse anti-p38 (clone A-12, 1:500; Biotechnologies, Santa Cruz, CA, USA) and an appropriate secondary Ab. 


\subsection{Flow Cytometry}

HBMEC cultured on six-well plates to subconfluency were stimulated as indicated and subsequently detached with Accutase ${ }^{\mathrm{TM}}$ (PAA Laboratories, Coelbe, Germany). Then they were washed and incubated with a FITC-labeled mouse anti-ICAM-1 antibody (clone BBIG-I1, R\&D Systems) or the corresponding FITC-labeled isotype control IgG1 (BD Biosciences, Heidelberg, Germany). Flow cytometry was performed on a FACSCalibur (BD Biosciences) fluorescence-activated cell sorter.

\subsection{Adhesion Assay}

Adhesion assays were performed exactly as previously described in detail [25]. Briefly, $5 \times 10^{4}$ HBMEC per well were seeded on 96-well plates and grown to confluency which usually took 48 to $72 \mathrm{~h}$. Subsequently, cells were incubated as indicated. After washing, calcein-labeled (Molecular Probes, Life Technologies), PMA-activated Jurkat T cells were added in FCS-free RPMI1640 medium for $30 \mathrm{~min}$ at $37{ }^{\circ} \mathrm{C}$. After additional washing steps, fluorescence intensity, reflecting the number of adherent cells in a linear manner, was determined using a Fluoroskan Ascent microplate fluorometer (Thermo Sientific, Waltham, MA, USA).

\section{Conclusions}

Based on previously published experimental results, it seems conceivable that FAE reduce T-cell infiltration into the CNS parenchyma of patients with MS. Our findings, however, suggest that this potential mode of action, if present, is not mediated by a direct effect of FAE on endothelial cells at the BBB under inflammatory conditions.

\section{Acknowledgments}

The authors are grateful to Svetlana Hilz for excellent technical assistance and to Guido Stoll for his support of this project. This study was financed by local University research funds from the State of Bavaria.

\section{Author Contributions}

Axel Haarmann designed, performed and supervised experiments, analyzed data and drafted the manuscript; Mathias Nehen performed experiments, analyzed data and approved the manuscript; Annika Deiß performed experiments, analyzed data and approved the manuscript; Mathias Buttmann conceived and designed the study, supervised experiments, analyzed data and edited the manuscript.

\section{Conflicts of Interest}

The authors declare no conflict of interest. 


\section{References}

1. Engelhardt, B.; Ransohoff, R.M. Capture, crawl, cross: The T cell code to breach the blood-brain barriers. Trends Immunol. 2012, 33, 579-589.

2. Buttmann, M.; Rieckmann, P. Treating multiple sclerosis with monoclonal antibodies. Expert Rev. Neurother. 2008, 8, 433-455.

3. Haarmann, A.; Nowak, E.; Deiss, A.; van der Pol, S.; Monoranu, C.M.; Kooij, G.; Muller, N.; van der Valk, P.; Stoll, G.; de Vries, H.E.; et al. Soluble VCAM-1 impairs human brain endothelial barrier integrity via integrin $\alpha$-4-transduced outside-in signalling. Acta. Neuropathol. 2015, 129, 639-652.

4. Brinkmann, V.; Billich, A.; Baumruker, T.; Heining, P.; Schmouder, R.; Francis, G.; Aradhye, S.; Burtin, P. Fingolimod (FTY720): Discovery and development of an oral drug to treat multiple sclerosis. Nat. Rev. Drug Discov. 2010, 9, 883-897.

5. Van Doorn, R.; Lopes Pinheiro, M.A.; Kooij, G.; Lakeman, K.; van het Hof, B.; van der Pol, S.M.; Geerts, D.; van Horssen, J.; van der Valk, P.; van der Kam, E.; et al. Sphingosine 1-phosphate receptor 5 mediates the immune quiescence of the human brain endothelial barrier. J. Neuroinflamm. 2012, 9, 133.

6. Schilling, S.; Goelz, S.; Linker, R.; Luehder, F.; Gold, R. Fumaric acid esters are effective in chronic experimental autoimmune encephalomyelitis and suppress macrophage infiltration. Clin. Exp. Immunol. 2006, 145, 101-107.

7. Linker, R.A.; Lee, D.H.; Ryan, S.; van Dam, A.M.; Conrad, R.; Bista, P.; Zeng, W.; Hronowsky, X.; Buko, A.; Chollate, S.; et al. Fumaric acid esters exert neuroprotective effects in neuroinflammation via activation of the Nrf2 antioxidant pathway. Brain 2011, 134, 678-692.

8. Treumer, F.; Zhu, K.; Glaser, R.; Mrowietz, U. Dimethylfumarate is a potent inducer of apoptosis in human T cells. J. Investig. Dermatol. 2003, 121, 1383-1388.

9. Gold, R.; Kappos, L.; Arnold, D.L.; Bar-Or, A.; Giovannoni, G.; Selmaj, K.; Tornatore, C.; Sweetser, M.T.; Yang, M.; Sheikh, S.I.; et al. Placebo-controlled phase 3 study of oral BG-12 for relapsing multiple sclerosis. N. Engl. J. Med. 2012, 367, 1098-1107.

10. Dehmel, T.; Dobert, M.; Pankratz, S.; Leussink, V.I.; Hartung, H.P.; Wiendl, H.; Kieseier, B.C. Monomethylfumarate reduces in vitro migration of mononuclear cells. Neurol. Sci. 2014, 35, 1121-1125.

11. Rubant, S.A.; Ludwig, R.J.; Diehl, S.; Hardt, K.; Kaufmann, R.; Pfeilschifter, J.M.; Boehncke, W.H. Dimethylfumarate reduces leukocyte rolling in vivo through modulation of adhesion molecule expression. J. Investig. Dermatol. 2008, 128, 326-331.

12. Wallbrecht, K.; Drick, N.; Hund, A.C.; Schon, M.P. Downregulation of endothelial adhesion molecules by dimethylfumarate, but not monomethylfumarate, and impairment of dynamic lymphocyte-endothelial cell interactions. Exp. Dermatol. 2011, 20, 980-985.

13. Vandermeeren, M.; Janssens, S.; Borgers, M.; Geysen, J. Dimethylfumarate is an inhibitor of cytokine-induced E-selectin, VCAM-1, and ICAM-1 expression in human endothelial cells. Biochem. Biophys. Res. Commun. 1997, 234, 19-23. 
14. Loewe, R.; Holnthoner, W.; Groger, M.; Pillinger, M.; Gruber, F.; Mechtcheriakova, D.; Hofer, E.; Wolff, K.; Petzelbauer, P. Dimethylfumarate inhibits TNF-induced nuclear entry of NF- $\kappa \mathrm{B} / \mathrm{p} 65$ in human endothelial cells. J. Immunol. 2002, 168, 4781-4787.

15. Kallmann, B.A.; Wagner, S.; Hummel, V.; Buttmann, M.; Bayas, A.; Tonn, J.C.; Rieckmann, P. Characteristic gene expression profile of primary human cerebral endothelial cells. FASEB J. 2002, 16, 589-591.

16. Voraberger, G.; Schafer, R.; Stratowa, C. Cloning of the human gene for intercellular adhesion molecule 1 and analysis of its 5 '-regulatory region. Induction by cytokines and phorbol ester. J. Immunol. 1991, 147, 2777-2786.

17. Abadier, M.; Haghayegh Jahromi, N.; Cardoso Alves, L.; Boscacci, R.; Vestweber, D.; Barnum, S.; Deutsch, U.; Engelhardt, B.; Lyck, R. Cell surface levels of endothelial ICAM-1 influence the transcellular or paracellular T-cell diapedesis across the blood-brain barrier. Eur. J. Immunol. 2015, 45, 1043-1058.

18. Venci, J.V.; Gandhi, M.A. Dimethyl fumarate (Tecfidera): A new oral agent for multiple sclerosis. Ann. Pharmacother. 2013, 47, 1697-1702.

19. Benardais, K.; Pul, R.; Singh, V.; Skripuletz, T.; Lee, D.H.; Linker, R.A.; Gudi, V.; Stangel, M. Effects of fumaric acid esters on blood-brain barrier tight junction proteins. Neurosci. Lett. 2013, $555,165-170$.

20. Kunze, R.; Urrutia, A.; Hoffmann, A.; Liu, H.; Helluy, X.; Pham, M.; Reischl, S.; Korff, T.; Marti, H.H. Dimethyl fumarate attenuates cerebral edema formation by protecting the blood-brain barrier integrity. Exp. Neurol. 2015, 266, 99-111.

21. Gumbleton, M.; Audus, K.L. Progress and limitations in the use of in vitro cell cultures to serve as a permeability screen for the blood-brain barrier. J. Pharm. Sci. 2001, 90, 1681-1698.

22. Omidi, Y.; Campbell, L.; Barar, J.; Connell, D.; Akhtar, S.; Gumbleton, M. Evaluation of the immortalised mouse brain capillary endothelial cell line, b.End3, as an in vitro blood-brain barrier model for drug uptake and transport studies. Brain Res. 2003, 990, 95-112.

23. Song, L.; Pachter, J.S. Culture of murine brain microvascular endothelial cells that maintain expression and cytoskeletal association of tight junction-associated proteins. In Vitro Cell. Dev. Biol. Anim. 2003, 39, 313-320.

24. Haarmann, A.; Deiss, A.; Prochaska, J.; Foerch, C.; Weksler, B.; Romero, I.; Couraud, P.O.; Stoll, G.; Rieckmann, P.; Buttmann, M. Evaluation of soluble junctional adhesion molecule-A as a biomarker of human brain endothelial barrier breakdown. PLOS ONE 2010, 5, e13568.

25. Buttmann, M.; Lorenz, A.; Weishaupt, A.; Rieckmann, P. Atorvastatin partially prevents an inflammatory barrier breakdown of cultured human brain endothelial cells at a pharmacologically relevant concentration. J. Neurochem. 2007, 102, 1001-1008.

26. Buttmann, M.; Berberich-Siebelt, F.; Serfling, E.; Rieckmann, P. Interferon-beta is a potent inducer of interferon regulatory factor-1/2-dependent IP-10/CXCL10 expression in primary human endothelial cells. J. Vasc. Res. 2007, 44, 51-60.

(C) 2015 by the authors; licensee MDPI, Basel, Switzerland. This article is an open access article distributed under the terms and conditions of the Creative Commons Attribution license (http://creativecommons.org/licenses/by/4.0/). 